\title{
PAULO FREIRE, ALFABETIZAÇÃO E LUTAS SOCIAIS NA AMÉRICA LATINA.
}

\author{
PAULO FREIRE, ALFABETIZACIÓN Y LUCHA SOCIAL EN AMÉRICA LATINA
}

\section{PAULO FREIRE, LITERACY AND SOCIAL STRUGGLES IN LATIN AMERICA}

\author{
Doi:https://doi.org/10.9771/gmed.v13i3.47457
}

\author{
Micaela Ovelar ${ }^{1}$ \\ Catherine Murphy²
}

\begin{abstract}
A Revista Germinal entrevistou Micaela Ovelar e Catherine Murphy, produtora e diretora respectivamente do documentário Fonemas da Liberdade ${ }^{3}$, recém lançado no Brasil e que tem como tema o Centenário de Paulo Freire. O documentário é uma das produções audivisuais do The Literacy Project ${ }^{4}$ que elas fazem parte e que também já produziu outros documentários como o Maestra ${ }^{5}$ que aborda a participação das mulheres na Campanha de Alfabetização de Cuba e Mi primera tarea ${ }^{6}$ que conta como Silvio Rodriguez, um dos artistas cubanos mais reconhecido mundialmente, também foi um alfabetizador dessa campanha quando era jovem.
\end{abstract}

Germinal: Gostaríamos de iniciar essa entrevista perguntando como vocês entendem essa relação entre as artes audiovisuais, educação e as lutas sociais? Como vocês têm desenvolvido isso no "The Literacy Project"?

Micaela Marquez e Catherine Murphy: Muchos de los movimientos sociales y, en general, las luchas sociales a lo largo de la historia han reivindicado el tema de la alfabetización y el derecho a la educación como una de sus banderas principales, puesto que su negación como un derecho humano universal ha estado en la base de las inequidades, inequidades muchas veces institucionalizadas. Desafortunadamente esto ha ocurrido en todo el mundo. Por eso, desde The Literacy Project hemos querido -a través de nuestro trabajo- poner el foco, visibilizar, celebrar, y dar a esas demandas sociales un papel protagónico. De allí que hayamos abarcado, trabajado y avanzado con el tema del acceso a la educación y la alfabetización. 
Tanto nuestro trabajo retratando la Campaña Nacional de Alfabetización en Cuba, como los nuevos trabajos, entre ellos, el cortometraje "Fonemas de Liberdade", que acaba de ser estrenado para público brasileño y que trata sobre las primeras experiencias pedagógicas de Paulo Freire; hablan de un aprendizaje, de una enseñanza eterna que el patrono de la educación brasileña ha dejado para el mundo, para todas aquellas personas que luchan por un mundo con mayor equidad y justicia social.

\section{G: Em que medida o documentário sobre o centenário de Paulo Freire está localizado nessa experiência de vocês?}

M.M e C.M: Sí, el legado de Paulo Freire está profundamente vinculado a todos los demás trabajos de The Literacy Project porque -como decíamos- abarcamos estos temas de educación y alfabetización, pero también mirando y apostando por procesos de educación emancipatoria, procesos de alfabetización y educación libertadora, de empoderamiento, que toman de base la metodología freireana. A estos conceptos, a los que Paulo Freire entregó su vida desde una filosofía que llama a la reflexión-acción, estamos profundamente vinculados, y son una guía fundamental en todo lo que hacemos.

Tal es así que para nosotros fue una especie de deber mayor crear una obra honrando el legado de Paulo Freire en un momento tan especial como el centenario de su nacimiento. Esta una idea, en realidad, la teníamos desde hace muchos años y siempre se nos hacía un poco difícil pensar en qué podíamos aportar algo nuevo, contribuir una película con una nueva perspectiva, algo fresco sobre una persona tan grande como Paulo Freire, tan celebrado, tan estudiado, tan retratado en el mundo entero.

No obstante, en la investigación decidimos enfocarnos en la parte que justamente se encuentra más vinculada a las otras producciones audiovisuales de The Literacy Project. Nos referimos a las primeras experiencias pedagógicas de Paulo Freire con base a la alfabetización de jóvenes y adultos. Freire cambió la forma de comprender la alfabetización, concientizando y co-creando una metodología con alumnos de Angicos y con los coordinadores, jóvenes estudiantes de la Universidad de Recife principalmente. Esa experiencia de Angicos, fue una base muy sólida en el trabajo de Freire, antes de la dictadura antes del golpe, de la dictadura y del exilio, incluso antes de publicar su primer libro, y sobre lo cual Freire fundamentó su vida en un constante reflexionando y evolucionando.

G: No governo Bolsonaro vemos constantemente Paulo Freire ser atacado, a partir da sua experiência com o documentário "Fonemas da Liberdade" como você vê a relevância e a atualidade da obra freiriana para educação brasileira?

M.M e C.M: Las perspectivas freireanas en el campo de la Educación, sea esta popular o tradicional, son por un lado esenciales, vitales, para reconstruir los lazos de las relaciones sociales, políticas, económicas... en una 
sociedad tan diversa, tan extensa, tan desigual, y, al mismo tiempo, tan rica culturalmente como lo es la sociedad brasileña. Justamente, por esto, es que la obra, la vida y el legado de Paulo Freire tienen, por otro lado, una actualidad y una vigencia que son innegables. De hecho, las experiencias pedagógicas que llegan a nuestros días de la mano del patrono de la educación brasileña, de su genuina filosofía de la Educación, son cada día más necesarias, ante la profundización de las desigualdades, las inequidades, en fin, ante la complejidad de las relaciones sociales.

Pero, claro, hay un sector minoritario pero muy poderoso de la sociedad y del estamento político brasileño, que se encuentra lamentablemente en este momento en el poder, que no solo no le importa las pedagogías de la liberación del ser humano históricamente oprimido, y menos que ese "romper las cadenas de la opresión" sea a través de la educación. Porque la Educación es el camino, no para resolver todos y cada uno de los problemas que aquejan a la sociedad, pero sí, para una parte muy considerable de esas deudas pendientes que el Estado tiene con la población más necesitada. En este sentido, es posible entender -sin jamás aceptar ni menos avalar- el odio y el rechazo que recibe el nombre de Paulo Freire y sus enseñanzas, que, sin embargo, nunca más podrán ser olvidadas.

G: A Revista Germinal: marxismo e educação em debate têm como eixo central acolher reflexões que abordem a educação a partir da rica tradição marxista, e nesse sentido, também recebemos muitos artigos que tratam das experiências socialistas. Como vocês percebem na vida e na obra de Paulo Freire essa relação com as experiências socialistas?

M.M e C.M Este es un tema en debate, por lo que hemos podido indagar en una primera instancia Paulo Freire no se identificaba ideológicamente con el socialismo ni con el comunismo, sino más bien con la filosofía de la liberación y el cristianismo. Sin embargo, su filosofía de la Educación, que creemos también era su propia filosofía de vida, sí se conciliaba muy bien con las prácticas que podrían transformar una sociedad a través de relaciones (de producción social) más justas y sin la apropiación de las clases burguesas de la plusvalía intelectual y cultural de las poblaciones más oprimidas.

Luego, humildemente nos parece que eso va cambiando en Freire, tal vez sus experiencias reales, las vivencias profundamente humanas que experimentó al ser invitado a contribuir en los programas de alfabetización y educación popular, de jóvenes y adultos, en los procesos emancipadores de África, Asia, América Latina, El Caribe... y, por supuesto, su propio vínculo reforzado por su ser, esencialmente nordestino, pernambucano, con los procesos políticos y con las realidades sociales que vivía -y aún vive- hoy Brasil. De manera, que en nuestro criterio al final de su maravilloso "andarilhagem" en este plano terrenal, Freire puede haber abrazado las experiencias socialistas, no somo como experiencias de cambios radicales en las conciencias de los seres humanos, sino ideológicamente, como bandera de luchas para la liberación humana en pleno vínculo con la vida y la naturaleza en todas sus formas. Pero claro, esto es una percepción totalmente subjetiva. 
G: No documentário, há o tratamento direto da reflexão de Paulo Freire com a Revolução Cubana. Como foi isso?

M.M e C.M: Es muy interesante que estas dos experiencias pedagógicas suceden casi en el mismo momento, en un mismo momento histórico. La Campaña Nacional de Alfabetización Cubana fue impulsada por una Revolución, que se instauró como sabemos en 1959, y que siempre tuvo como prioridad la educación. En la década de 1960, Freire ya se encontraba trabajando con la concepción de los primeros Círculos de Cultura y comenzando a aplicar sus teorías pedagógicas. Una de las grandes interrogantes que no hemos podido dilucidar aún es saber hasta qué punto Freire pudo haber estado al tanto de lo que pasaba en Cuba y hasta qué punto eso pudo haberlo influenciado, y en qué formas, pero lo que ha quedado son las luchas sociales para enfrentar el masivo analfabetismo como un campo de lucha fundamental.

En la plataforma de la revolución cubana el acceso a la educación fue un pilar fundamental, eso es indiscutible, porque en la Cuba del aquel momento, de los 6 millones de habitantes que tenía la isla, 1 millón era analfabeta según los censos de la década de 1950. Por otra parte, en el nordeste de Brasil, al menos lo que se plantea en el documental histórico As 40 horas de Angicos, alrededor de 75\% de las personas eran iletradas y no podían votar. Por ser analfabetos tenían prohibido ejercer el derecho al sufragio.

G. Vocês também produziram o documentário "Mi primera tarea" que trata da participação do cantor Silvio Rodriguez na Campanha de Alfabetização de Cuba. Silvio Rodriguez é considerado um dos maiores artistas cubanos e sempre foi uma pessoa ativa nos debates e desafios da construção do socialismo em Cuba. Isso aparece no documentário? Como?

M.M e C.M Nuestro documental "Silvio Rodríguez: Mi Primera Tarea" justamente retrata la vivencia, el testimonio del amado cantautor cubano, quien cuenta en primera persona todo sobre su participación en la Campaña Nacional de Alfabetización Cubana.

Silvio tenía apenas 14 años en aquel entonces. Era un joven, un adolescente como muchos otros. En la Campaña cubana participaron 100 mil jóvenes de menos de 18 años que dedicaron un año de su vida en esa loable labor. Podemos decir que Silvio Rodríguez dedicó una buena parte del año 1961 a compartir lo que él había aprendido en la escuela, con sus compatriotas que no habían tenido oportunidad de estudiar. Entonces, nosotros en la realización de la película tuvimos la bendición, el honor de poder grabar el testimonio de Silvio, gracias a nuestros amigos, compañeros, productores y coproductores de aquel proyecto, entre ellos, el Centro Martin Luther King de La Habana, el gran fotógrafo cubano Roberto Chile, quien grabó mucho de los testimonios, bueno, tenemos como 80 testimonios grabados de los más jóvenes alfabetizadores que participaron en la epopeya de la Campaña Cubana de 1961. Silvio es uno de ellos, y claro, siendo un poeta, una figura tan amada en el mundo de habla castellana, con un compromiso social muy sólido en dentro y fuera de la sociedad 
cubana, ha sido un gran honor que él nos cuente en primera persona sobre su vivencia alfabetizando, que -valga decir- fue su primera acción social y fue un poco el comienzo de su camino también.

Este año 2021, este mes de diciembre, ahora, estamos en la víspera de celebrar el 60 aniversario de la Campaña Nacional de Alfabetización cubana entonces estamos también comprometiéndonos con una colaboración con varios jóvenes creadores en La Habana, incluyendo el Dúo Obsesión qué hace educación popular a través del eje óptico de la concientización y la educación popular. Estamos sacando una serie de pequeñas cápsulas del mar de testimonios que habíamos grabado en los últimos 10 años, capsulas de 2-3 minutos, que les invitamos que puedan ver, que disfruten mucho y que son parte de una gran obra colectiva.

\section{G: Que semelhanças e diferenças vocês conseguiram perceber entre a Campanha Nacional de Alfabetização em Cuba e a experiência de Angicos?}

M.M e C.M Las diferencias con Angicos, yo creo que hay diferencias pedagógicas y metodológicas. Pero lo que más me viene a la mente es que la experiencia de Angicos fueron alrededor de 300 alumnos y alumnas, era un programa piloto, con deseos de que se aplicara a nivel nacional, pero esto fue violentamente interrumpido por la dictadura militar. Mientras que, en Cuba, la Campaña de Alfabetización fue planificada por la Revolución, tuvo un alcance nacional, fue un programa nacional, contó con una comisión pedagógica, donde resaltaba como una de las figuras clave Raúl Ferrer, a quien me lo han descripto como el Paulo Freire cubano, Ferrer fue un educador popular en la Cuba de la década de 1950 y en adelanta. Cuando yo empecé mi investigación él ya había fallecido lamentablemente nunca lo pude conocer en persona. Pero, bueno, en Cuba ellos hicieron también un censo, luego los alfabetizadores fueron recibidos en las comunidades de los alfabetizandos, compartían con sus alumnos en sus casas. Yo no sé si eso mismo pasó en Angicos, por ejemplo, yo no sé dónde vivieron los estudiantes de Recife, donde se quedaron los coordinadores durante todo el tiempo que estuvieron en Angicos, y más detalles sobre su interacción con las personas locales, esos detalles no los conozco de la experiencia en Angicos.

En resumen, en Cuba fue nacional, fue algo masivo, era la prioridad de la nación en esos años del comienzo de la Revolución Cubana, ellos se fijaron la meta de alfabetizar a un millón de personas, hicieron un llamado a toda la población a participar y de hecho participaron adultos y jóvenes, maestros retirados, amas de casa, recién graduados que no estaban trabajando todavía. Se movilizaron a los sindicatos, las organizaciones sociales como la Federación de Mujeres, ellos movilizaron a todos y valga la redundancia del verbo movilizar, pero toda la sociedad, la sociedad entera se movilizó para dar ese gran primer paso de transformar a la nación lo que fue decir: "ahora la educación es para todos y todas".

G: No mesmo contexto do início da Revolução Cubana em que foi desenvolvido a Campanha de Alfabetização, foram criadas várias iniciativas e instituições no âmbito educacional e cultural. O 
cinema teve, e ainda persiste, um papel de destaque com a criação do Instituto Cubano de Arte e Indústria Cinematográfica (ICAIC). Como vocês avaliam essa relação entre o cinema e a luta pelo socialismo, em especial, na América Latina?

M.M e C.M: Sí, claro, desde el momento que la revolución cubana toma el poder, luego del triunfo de enero de 1959, se comienza a transformar profundamente toda la sociedad en todos los ámbitos con el propósito de construir un sistema social e inclusivo. La educación es para todos y todas, la salud es para todos y todas, la vivienda es para todos y todas, no solo había que construir un sistema nacional de educación, sino de salud, aprobar la reforma agraria, nacionalizar los latifundios, nacionalizar las compañías de electricidad y mucho más, existía la United Fruit Company etcétera y también comenzaron a socializar la cultura para todos y todas, creando el ICAIC, el instituto de cine para también contar sus propias historias: muchos de los cines en Latinoamérica de los años 1950 la programación venía desde el norte, desde la perspectiva gringa o norteamericana, "del norte" como decían ellos y entonces era también preciso transformar eso. Realmente, de ese contexto, nace la gran obra de Santiago Álvarez con los Noticieros Latinoamericanos, que tenemos también el gran honor y bendición de haber podido utilizar -con asesoramiento de la Oficina de Santiago Álvarez- en muchas de nuestras obras documentales.

G: O Brasil vivenciou recentemente o episódio de destruição por incêndio de um galpão da Cinemateca Brasileira. O espaço continha um conjunto de materiais que possuíam grande importância para preservação, memória e difusão da produção cinematográfica brasileira. Diversos grupos viram nessa situação a expressão de uma política deliberada de descaso do governo Bolsonaro na lógica capitalista e neoliberal. Como vocês têm analisado essa questão no contexto do capitalismo na América Latina? Qual a importância desse debate para se pensar um projeto de sociedade que supere a lógica do capital?

M.M e C.M En primer lugar, quisiéramos expresar nuestra solidaridad con todos los cineastas, guionistas, actores, actrices, en fin, artistas y cultores en general, que han perdido sus obras audiovisuales en el incendio de la Cinemateca Brasileira. Es una tragedia no solo por las pérdidas materiales sino sobre todo por las pérdidas inmateriales. Se trata de un reservorio muy importante de la historia y de la cultura brasileña. Es triste e indignante al mismo tiempo. Si bien, realmente, no conocemos los detalles de lo que sucedió en la Cinemateca Brasileira, ni cómo se han desarrollado las investigaciones, sí podríamos aportar en relación a la importancia de la preservación de la cultura y la historia, en especial, la cultura no enajenada que proviene del pueblo, la captación audiovisual de la memoria oral, las tradiciones ancestrales, pero también son importantes las nuevas expresiones culturales y que está la sociedad queriendo transmitir con ellas.

En ese sentido, recordar a Antonio Gramsci cuando hablaba de la hegemonía del capitalismo, que va más allá de las relaciones económicas de poder, es decir, que también ha infectado las culturas, de modo que la 
batalla de ideas nos lleva irremediablemente al campo cultural. Nos lleva a la defensa de la cultura desde lógicas anti capitalistas y a la construcción de otros modos de relaciones sociales, que pongan en el centro al ser humano, a la naturaleza y no al extractivismo, a la esclavitud, a la violación de los derechos básicos de las personas. El objetivo del neoliberalismo siempre ha sido la acumulación exponencial de las ganancias capitalistas, sin importar que medios se utilicen para obtener plusvalía. En ese sentido, la Revolución Cultural nos debe llevar a proteger, defender y difundir todas las formas de culturales contrahegemónicas.

La pérdida de esos materiales cinematográficos es sin duda invaluable. Sabemos que los gobiernos neoliberales, de derecha extrema y neoconservadores, no tienen ningún respeto por la cultura, de hecho, los primeros recortes que realizan este tipo de gobiernos es a los presupuestos de Educación, Cultura y Salud, por ello, nos solidarizamos con el pueblo brasileño y esperamos de corazón que se pueda recuperar algunos de los materiales cinematográficos, así como reedificar la Cinemateca Brasileira para seguir dando la lucha cultural, en el Brasil y en el mundo.

G: Micaela, tú, en particular, también trabajaste con el presidente Hugo Chávez, quien debatió la necesidad de construir el socialismo y llevó a cabo el proyecto de Revolución bolivariana. ¿Cuál es tu evaluación de la importancia de la educación y también de las artes en esta reflexión en el contexto venezolano?

M.M: El presidente Hugo Chávez otorgó a la Educación un papel fundamental en su proyecto político revolucionario, la educación estuvo presente desde su concepción germinal hasta su concreción real, y, luego, durante todo el desarrollo de sus mandatos presidenciales. En 1991, un aún desconocido Chávez escribió "El libro azul" allí diseñó las líneas estratégicas de su programa político, el cual en 1998 lo llevó a ganar las elecciones presidenciales democráticamente y pocos meses después, ya en 1999, propició la refundación de la República Bolivariana de Venezuela.

Es importante señalar que uno de los pilares del pensamiento filosófico político de Hugo Chávez lo encontramos en Simón Rodríguez (conocido también como Samuel Robinson, un seudónimo o nombre de guerra). Rodríguez fue maestro del Libertador Simón Bolívar, quien se refería a él como "El Sócrates de Caracas" por su estilo dialógico de enseñanza. De hecho, fue Simón Rodríguez quien propone la "utopía" de un nuevo Estado para Venezuela, que en aquel momento luchaba por emanciparse de la Corona española. Dos siglos más tardes, Chávez revive el sueño de un país soberano, de la mano de la educación liberadora, la soberanía y la independencia de Venezuela. El presidente Chávez lo llamó "la utopía concreta robinsoniana".

Es decir, el mandatario venezolano siempre fue consciente de que el proyecto bolivariano se sostendría sobre las bases de la Educación, en sentido amplio, popular, escolar, académica, escénicas, musicales, artísticas. Todas las formas, maneras y niveles eran necesarios y vitales para el futuro de la Revolución Bolivariana. Fue así que en este siglo XXI, creó las misiones educativas y desarrolló las artes, entre otras políticas 
públicas inclusivas, democratizadoras de los saberes y de la cultura popular venezolana. Dar un lugar destacado, estelar diría yo, a la Educación es una de las características principales que podemos encontrar en todos los procesos revolucionarios genuinos, así como en aquellos líderes y lideresas de todos los tiempos. Y, por ello, no podía estar ausente en la Revolución Bolivariana.

\section{G. Palavras da diretora Catherine Murphy}

C.M: Para cerrar la entrevista, quisieras decir que en The Literacy Project hacemos estas obras para seguir cultivando, para seguir construyendo el otro mundo posible. Queremos honrar la historia de los movimientos sociales que han dado la lucha por la emancipación no solo de las naciones latinoamericanas y caribeñas, sino del ser humano en comunión con la naturaleza.

Esperamos que disfruten de nuestra película "Fonemas de Liberdade", así como también de otras producciones, las cuales están abiertas y disponibles en nuestra página web: www.theliteracyproject.org y en nuestro canal de youtube www.youtube.com/c/LiteracyProjectFilms . Allí van a poder conocer más de nuestro proyecto. También -además de ver los documentales- invitamos a otros educadores y educadoras a utilizar las realizaciones audiovisuales en las aulas u otras actividades. Nos haría muy felices compartir nuestros trabajos con ustedes y, al mismo tiempo, nos ayudarían mucho con la difusión de estos materiales educacionales. Muchas gracias!

\footnotetext{
Notas

1 Professora da Universidade Bolivariana da Venezuela (UBV) do curso Estudos Políticos e Governo e do curso de Relações Internacionais. Possui graduação em Estudos Políticos e Governo UBV (2007- 2011); Diploma em Estudos de Gênero - Centro de Estudos da Mulher - Universidade Central da Venezuela (UCV, 2011); Mestria em Relações Internacionais UCV (2013-2015). Mestria em Relações Internacionais, Instituto de Estudos Superiores de Defesa Nacional - Universidade Militar da Venezuela (UMBV, 20132014). Especialista em Direção de Cinema e TV - Escola de Meios de Produção Audiovisual (2011-2013). Integra o Grupo de Estudos Estado e Protestos Sociais do Centro de Estudos em Economia Politica CEEP da Universidade Bolivariana da Venezuela; Grupo de Estudos e Pesquisas Movimentos Sociais e Educação do Campo e Cidade GEPEMDECC da Universidade Estadual do Sudoeste da Bahia (UESB); e também integra o Equipe Editorial da revista Práticas Educativas, Memórias e Oralidades REVPEMO da Universidade Estadual do Ceará, Fortaleza, Brasil EdUECE. Currículo lattes: http://lattes.cnpq.br/5383434865728130 ORCID: https://orcid.org/0000-0002-4266-8717 e-mail: micaela.ovelar@gmail.com

2 Maestra em Desarollo Social x FLACSO. Realizadora y fundadora de The Literacy Project (O Proyecto Alfabetizador) 3https://www.youtube.com/watch?v=GaxvjhSl7rU

4https://theliteracyproject.org/

5Para assistir o documentário Maestra com legenda em português acesse: https://youtu.be/CbEW9KsFmeI 6 https://www.youtube.com/watch?v=ug1G7dN6PJg
} 